\title{
Chiral symmetry, di-electrons and charm
}

\author{
Burkhard KAMPFER* ${ }^{* \dagger}$ \\ FZ Dresden-Rossendorf, 01314 Dresden, Germany \\ E-mail: kaempferdfzd.de
}

Thomas HILGER, Henry SCHADE, Robert SCHULZE

FZ Dresden-Rossendorf, 01314 Dresden, Germany

E-mail: t.hilgerefzd.de L.schadedfzd.de, F.schulzedfzd.de

\section{Gyorgy WOLF}

KFKI RMKI, H-1525 Budapest, POB 49, Hungary

e-mail: woLfdrmki.kfki.hu

\begin{abstract}
We survey some prospects of identifying further in-medium modifications of hadrons in a strongly interacting medium with respect to ongoing experiment series of the HADES Collaboration and planned experiments of the CBM Collaboration at FAIR. Di-electrons, strange and charm mesons are considered and their potential for signaling imprints of chiral restoration is highlighted.
\end{abstract}

XLVIII International Winter Meeting on Nuclear Physics in Memoriam of Ileana Iori 25-29 January 2010

Bormio, Italy

*Speaker.

${ }^{\dagger}$ Also at TU Dresden, 01062 Dresden, Germany. 


\section{Introduction}

In-medium hadron spectroscopy aims at quantifying the modifications of spectral functions of hadrons embedded in a strongly interacting medium. The Brown-Rho scaling prediction (cf. [U, [2] for recent surveys on this topic and further references therein) emphasized the tight relation of hadron masses and the chiral condensate, however, a strong impact of other condensates is conceivable (e.g., the poorly known four-quark condensates [ [3], 团] or the gluon condensates [0, 囵]). Due to the direct vector meson $(V)$ decays $V \rightarrow \gamma^{*} \rightarrow e^{+} e^{-}, \mu^{+} \mu^{-}$a measurement of the spectral function becomes feasible. Until now, broadening effects of the $\rho$ meson have been verified in cold nuclear matter $[\square]$ and in hot nuclear matter, see [[]]. From a measurement of the transparency ratio a strong broadening of the $\omega$ meson in cold nuclear matter is deduced [ $[8]$.

With respect to the ongoing experiment series of the HADES Collaboration and the planned experiments by the Compressed Baryon Matter Collaboration at FAIR we report here prospects for identifying medium modifications in the di-electron and strange/charm meson channels.

\section{Di-electrons}

Figure $\square$ (left panel) exhibits the di-electron spectrum as a function of the invariant mass for collisions $\mathrm{C}(2 \mathrm{AGeV})+\mathrm{C}$ (for details of the transport model of BUU type cf. [可). The solid black curve is for the superposition of $\pi^{0}, \eta$ and $\Delta$ Dalitz decays and bremsstrahlung as well as the separately displayed vacuum spectral functions of $\rho$ and $\omega$, while the dashed magenta curve depicts the modifications for in-medium spectral functions including collision broadening and extreme mass shifts by $-100 \mathrm{MeV}$ and $-50 \mathrm{MeV}$ (cf. [ए]] for corresponding QCD sum rule evaluations and the impact of quark condensates), respectively. Despite the assumed very drastic medium modifications, there is only a tiny imprint in the spectrum since the small system is too short living, with spectral functions evolving rapidly to the vacuum ones.

The prospects for verifying medium modifications are better for larger nuclei, as depicted in the right panel of Fig. 1 for $\mathrm{Au}(2 \mathrm{AGeV})+\mathrm{Au}$. The high-compression stage lasts significantly longer signaled by the stronger contribution of "shining" vector mesons. There is no indication of a double-hump $\omega$ signal caused by the shining in-medium mass-shifted contribution and the final vacuum decays in both cases considered.

In contrast to the data [ㅁ] for the reaction $\mathrm{C}(2 \mathrm{AGeV})+\mathrm{C}$, the data [ए2] for the reaction $\mathrm{C}(1 \mathrm{AGeV})+\mathrm{C}$ require a strong nucleon-nucleon bremsstrahlung, as pointed out in [ए3]]. In fact, the estimates in [14, [15] within the one-boson exchange model point to a strong bremsstrahlung contribution in proton-neutron collisions at $1 \mathrm{GeV}$ which is over-shined by the $\Delta$ Dalitz yield at higher beam energies.

\section{Open strange and charm mesons in nuclear matter}

In a schematic manner, in-medium modifications of hadrons may be encoded in effective masses, e.g. parameterized as $m^{*}=m_{0}+U n / n_{0}$, where $m_{0}$ stands for the vacuum mass and the interaction is condensed into the "potential" $U$, which can be related to the real part of the selfenergy. The dependence on the net baryon density $n$ can be involved; usually the linear density 

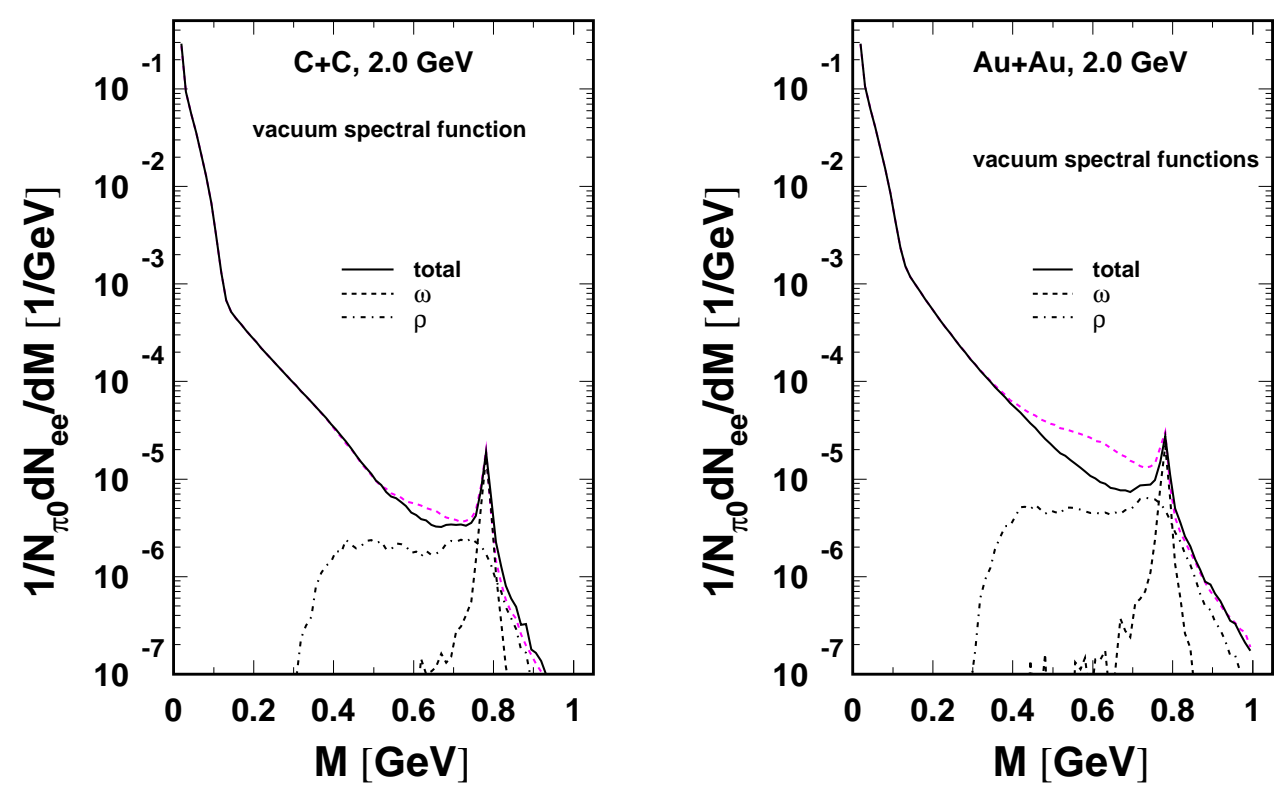

Figure 1: Invariant $e^{+} e^{-}$mass spectra in collisions $\mathrm{C}(2 \mathrm{AGeV})+\mathrm{C}$ (left panel) and $\mathrm{Au}(2 \mathrm{AGeV})+\mathrm{Au}$ (right panel). The solid curves employ the vacuum spectral functions of $\rho$ (dot-dashed curves) and $\omega$ (dotted curves), while the dashed magenta curves include in-medium effects. The contributions of $\pi^{0}, \eta$ and $\Delta$ Dalitz decays and bremsstrahlung are not depicted separately. From [0].

approximation around saturation density $n_{0}$ is employed. Figure $\square$ exhibits two examples for the transverse momentum distribution of $K^{+}$and $K^{-}$in collisions of $\operatorname{Ar}(1.756 \mathrm{AGeV})+\mathrm{KCl}$ calculated in a transport model of BUU type, cf. [ए]]. Clearly, an attractive (repulsive) net interaction with surrounding nucleons translates into a negative (positive) potential which enhance (deplete) the respective anti-kaon (kaon) abundances and modify the phase space distribution as seen in Fig. $\square$. Precision data for transverse momenta $p_{t} \sim 200 \mathrm{MeV} / \mathrm{c}$ in various rapidity bins, in particular at mid-rapidity, are needed to pin down reliably the actual values of the potentials.
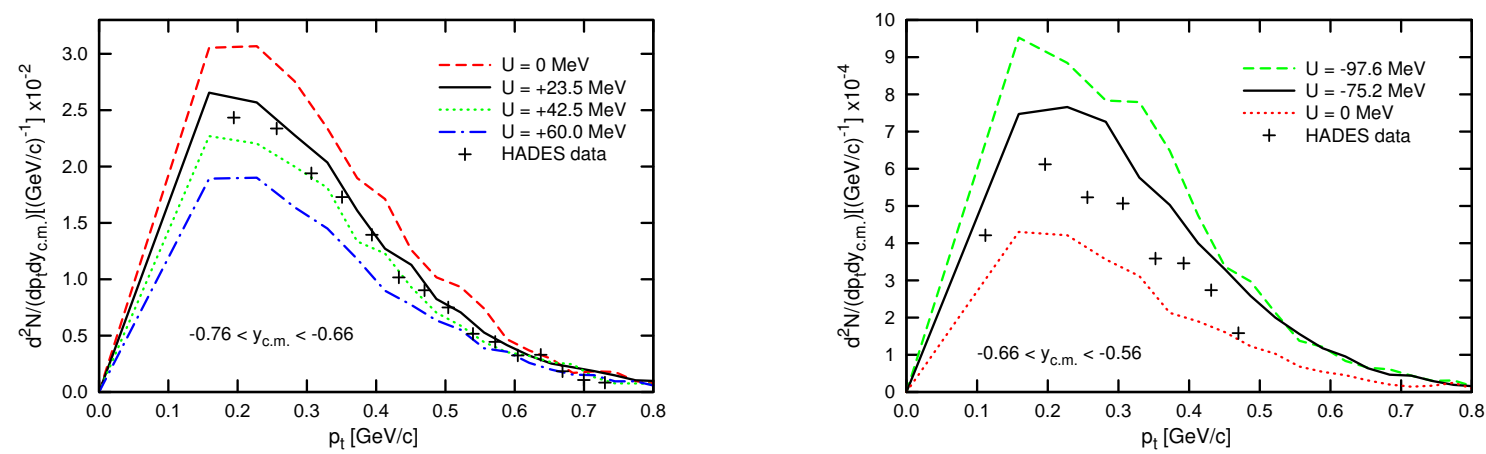

Figure 2: Transverse momentum distribution of kaons (left panel, rapidity interval $-0.76<y_{\text {c.m. }}<-0.66$ ) and anti-kaons (right panel, rapidity interval $-0.66<y_{c . m} .<-0.56$ ) for several values of the potential $U$ as indicated by the labels. All other settings of the BUU model [ए6] are frozen in. Data from [एव].

The calculation of $K^{ \pm}$spectral functions, from which the potential $U$ can be deduced, may be performed within hadronic interaction approaches, such as in [ए], [18], or may rely on QCD sum 
rules. The evaluation for $D^{ \pm}$mesons, for example, is based on the current-current correlator $\Pi(q)=$ $i \int d^{4} x e^{i q x}\left\langle\left\langle\mathscr{T}\left[j(x) j^{\dagger}(0)\right]\right\rangle\right\rangle$ with $j(x)=i \bar{d} \gamma_{5} c$ being the pseudo-scalar flavor changing current, $\mathscr{T}$ the time ordering operator, and $\langle\langle\cdots\rangle\rangle$ denotes the Gibbs average. Applying the operator-product expansion (OPE) at large squared momenta $q_{0}^{2}=-Q^{2}$ relates the correlator to QCD condensates. On the other hand, using a Borel transformed in-medium dispersion relation in the complex energy plane maps the even $(e)$ and the odd $(o)$ part of the OPE to moments of the spectral density $\Delta \Pi(s)$

$$
\begin{aligned}
& e \equiv \int_{s_{0}^{-}}^{s_{0}^{+}} d s s \Delta \Pi \mathrm{e}^{-s^{2} / M^{2}}=m_{+} F_{+} \mathrm{e}^{-m_{+}^{2} / M^{2}}+m_{-} F_{-} \mathrm{e}^{-m_{-}^{2} / M^{2}}, \\
& o \equiv \int_{s_{0}^{-}}^{s_{0}^{+}} d s \Delta \Pi \mathrm{e}^{-s^{2} / M^{2}}=F_{+} \mathrm{e}^{-m_{+}^{2} / M^{2}}-F_{-} \mathrm{e}^{-m_{-}^{2} / M^{2}},
\end{aligned}
$$

where we employed a pole ansatz $\Delta \Pi(s)=\pi F_{+} \delta\left(s-m_{+}\right)-\pi F_{-} \delta\left(s+m_{-}\right)$, which separates the perturbative continuum from the low-lying states by the threshold parameters $s_{0}^{ \pm} ; M$ is the Borel parameter. For $m_{ \pm}=m \pm \Delta m$ and $F_{ \pm}=F \pm \Delta F$ being independent of the Borel mass, $F_{ \pm}$can be eliminated, and the mass splitting and mass centroid of $D^{-}$and $D^{+}$are given by

$$
\Delta m=\frac{1}{2} \frac{o e^{\prime}-e o^{\prime}}{e^{2}+o o^{\prime}}, \quad m=\sqrt{\Delta m^{2}-\frac{e e^{\prime}+\left(o^{\prime}\right)^{2}}{e^{2}+o o^{\prime}}} .
$$

The strengths can then be obtained by $F_{ \pm}=e^{(m \pm \Delta m)^{2} / M^{2}}[e \pm(m \mp \Delta m) o] /(2 m) ; F$ and $\Delta F$ can be written in terms of the mass splitting and mass centroid as

$$
\begin{array}{r}
\Delta F=\frac{1}{2} \frac{\mathrm{e}^{\left(m^{2}+\Delta m^{2}\right) / M^{2}}}{m}\left[(e-o \Delta m) \sinh \left(\frac{2 m \Delta m}{M^{2}}\right)+o m \cosh \left(\frac{2 m \Delta m}{M^{2}}\right)\right], \\
F=\frac{1}{2} \frac{\mathrm{e}^{\left(m^{2}+\Delta m^{2}\right) / M^{2}}}{m}\left[(e-o \Delta m) \cosh \left(\frac{2 m \Delta m}{M^{2}}\right)+o m \sinh \left(\frac{2 m \Delta m}{M^{2}}\right)\right]
\end{array}
$$

which are exhibited in Fig. B. One can show that $F_{ \pm}\left(M_{0}\right)$ are extremal if $m_{ \pm}\left(M_{0}\right)$ are extremal, which allows for a consistent evaluation reported in [지. The found splitting of masses of $D^{+}$and $D^{-}$at non-zero baryon density corresponds to the splitting of $K^{-}$and $K^{+}$. It should be emphasized that up to mass-dimension six the density dependence of the condensates $m_{c}\langle\bar{d} d\rangle,\left\langle d^{\dagger} d\right\rangle,\left\langle d^{\dagger} D_{0}^{2} d\right\rangle$, and $\left\langle\frac{\alpha_{s}}{\pi} G^{2}\right\rangle$ drive the in-medium change of the $D$ mesons. As pointed out in [R]], the chiral condensate $\langle\bar{d} d\rangle$ in combination with the charm quark mass $m_{c}$ is the leading term for difference sum rules of chiral partners with the quark structure $q Q$, i.e. a light quark $(q)$ and a heavy quark $(Q)$, such as for $D$ meson states. That means, the difference of spectral moments, e.g. in the pseudoscalar - scalar or vector - axial-vector channels, is diminished with dropping modulus of the chiral condensate which may serve as order parameter of chiral restoration.

\section{Condensates at high temperature}

To employ QCD sum rules near the deconfinement transition one has to go beyond the lowdensity and/or low-temperature expansion. Following [[1, 目] the gluon condensate can be derived 

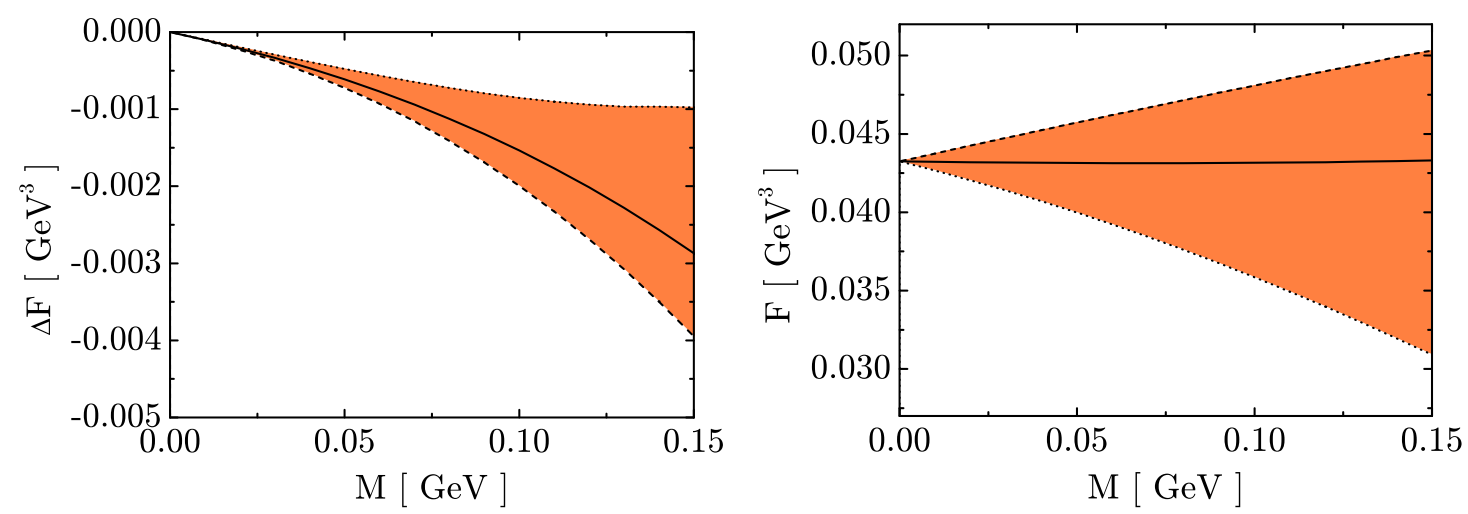

Figure 3: The quantities $\Delta F$ (left panel) and $F$ (right panel) entering the QCD sum rule for $D$ mesons. Line codes as in [20]].

from the interaction measure $e-3 p$, determined by the QCD trace anomaly, and the equation of state, which relates the energy density $e$ and the pressure $p$, as

$$
\begin{aligned}
& \left\langle\frac{\alpha_{s}}{\pi} G^{2}\right\rangle=\left\langle\frac{\alpha_{s}}{\pi} G^{2}\right\rangle_{0}-\frac{8}{9}(e-3 p), \\
& \left\langle\frac{\alpha_{s}}{\pi}\left((u G)^{2}-\frac{G^{2}}{4}\right)\right\rangle=-\frac{3}{4} \frac{\alpha_{s}}{\pi}(e+p),
\end{aligned}
$$

where the subscript 0 refers to the vacuum value. Contributions from light quarks to (4.]) are omitted in order to focus on the continuation to finite net baryon densities.

The presently most trusted values for the interaction measure and the equation of state at vanishing density (or equivalently quark chemical potential $\mu$ ) are results of Monte Carlo lattice simulations [22]. They can be extrapolated to finite chemical potential using a self-consistent quasi-particle model [23, 24]].
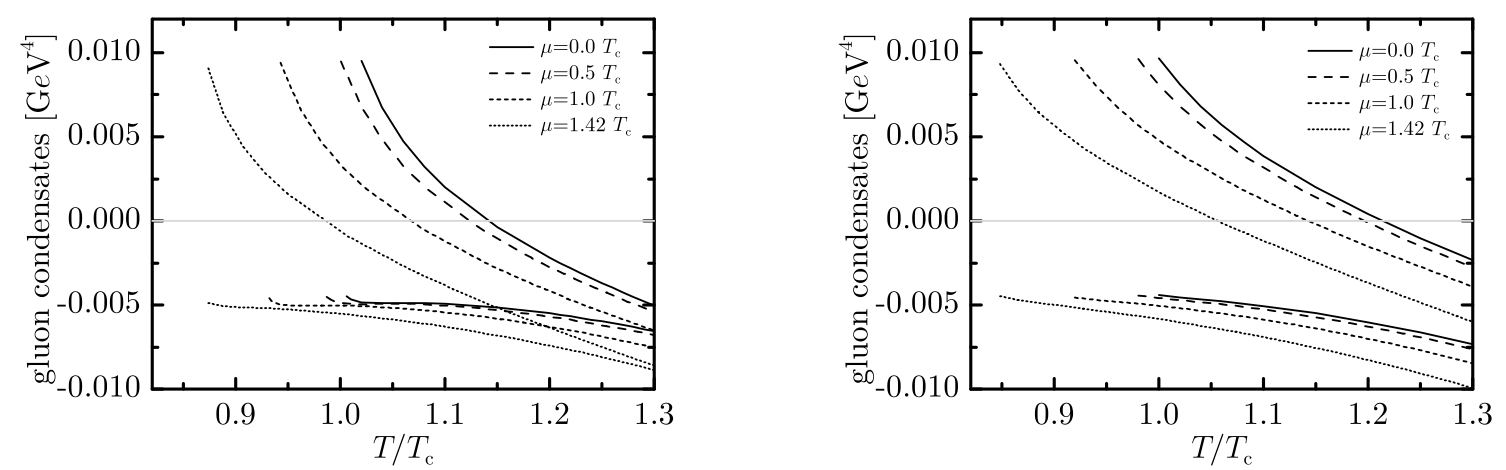

Figure 4: Gluon condensates (4.]) (upper curves) and (4.2) (lower curves) as a function of the temperature $T$ for several values of the quark chemical potential $\mu$ (see keys). The employed quasi-particle model is adjusted to recent lattice calculations [22]. The left (right) panel shows results for lattice temporal extent $N_{\tau}$ $=6$ (8). The critical temperature has been fixed to $T_{c}=190 \mathrm{MeV}$.

There is still some uncertainty about the continuum extrapolation of such lattice calculations. The current results were obtained on lattices with temporal extent $N_{\tau}$ of 6 and 8. Adjusting the 
quasi-particle model to both cases and employing the resulting values for $e$ and $p$ give the gluon condensates as exhibited in Fig. 田. Both condensates are monotonically decreasing functions of the temperature $T$. All curves start at the presumed locus of the phase transition, i.e. the boundary of the quasi-particle model determined by the characteristic curve (cf. [23] ) emerging from $T=T_{c}$ at $\mu=0$. Numerically, a scaling law $X\left(\mu / T_{c}=T(\mu=0) / T_{c}-0.045 T / T_{c}-0.027\left(T / T_{c}\right)^{2}\right)=$ const is observed for $X$ being one of the condensates (4.D) and (4.2). Additionally, both gluon condensates have a steeper slope for smaller $N_{\tau}$ with the difference being about the same size as the difference in the interaction measure of the lattice calculation leading to the assumption that future improved lattice results might lead to flatter curves. The condensates considered here determine, to a large extent, the behavior of $J / \psi$ near the deconfinement region.

\section{Summary}

In summary we emphasize that (i) large systems are required to enhance chances for verifying vector meson modifications in the di-electron channel in heavy-ion collisions at SIS18 energies. (ii) Phase space distributions of kaons and anti-kaons are sensitive to mass shifts encoded in interaction potentials. (iii) $D$ mesons exhibit a strong coupling to the chiral condensate and make them useful probes of chiral symmetry restoration imprints. (iv) A few QCD condensates are accessible at temperatures and baryon densities in the deconfinement transition region.

Acknowledgments: The work is supported by T71989 (Hungary), BMBF 06DR9059 and GSIFE (Germany).

\section{References}

[1] S. Leupold, V. Metag and U. Mosel, arXiv:0907.2388 [nucl-th].

[2] R. Rapp, J. Wambach and H. van Hees, arXiv:0901.3289 [hep-ph].

[3] R. Thomas, S. Zschocke and B. Kampfer, Phys. Rev. Lett. 95, 232301 (2005)

[4] R. Thomas, T. Hilger and B. Kampfer, Nucl. Phys. A 795, 19 (2007)

[5] K. Morita and S. H. Lee, Phys. Rev. C 77, 064904 (2008)

[6] K. Morita and S. H. Lee, Phys. Rev. Lett. 100, 022301 (2008)

[7] M. H. Wood et al. [CLAS Collaboration], Phys. Rev. C 78, 015201 (2008)

[8] M. Kotulla et al. [CBELSA/TAPS Collaboration], Phys. Rev. Lett. 100, 192302 (2008)

[9] H. W. Barz, B. Kampfer, G. Wolf and M. Zetenyi, arXiv:0910.1541 [nucl-th]

[10] S. Zschocke, O. P. Pavlenko and B. Kampfer, Eur. Phys. J. A 15, 529 (2002)

[11] G. Agakichiev et al. [HADES Collaboration], Phys. Rev. Lett. 98, 052302 (2007)

[12] G. Agakishiev et al. [HADES Collaboration], Phys. Lett. B 663, 43 (2008)

[13] E. L. Bratkovskaya and W. Cassing, Nucl. Phys. A 807, 214 (2008)

[14] L. P. Kaptari and B. Kampfer, Nucl. Phys. A 764, 338 (2006)

[15] L. P. Kaptari and B. Kampfer, Phys. Rev. C 80, 064003 (2009) 
[16] H. Schade, G. Wolf and B. Kampfer, Phys. Rev. C 81, 034902 (2010)

[17] M. Lutz, Phys. Lett. B 426, 12 (1998)

[18] M. F. M. Lutz, C. L. Korpa and M. Moller, Nucl. Phys. A 808, 124 (2008)

[19] G. Agakishiev et al. [HADES Collaboration], Phys. Rev. C 80, 025209 (2009)

[20] T. Hilger, R. Thomas and B. Kampfer, Phys. Rev. C 79, 025202 (2009)

[21] T. Hilger and B. Kampfer, arXiv:1001.0522 [nucl-th].

[22] A. Bazavov et al., Phys. Rev. D 80, 014504 (2009)

[23] R. Schulze, M. Bluhm and B. Kampfer, Eur. Phys. J. ST 155, 177 (2008)

[24] R. Schulze and B. Kampfer, Prog. Part. Nucl. Phys. 962, 386 (2009) 